\title{
Papers
}

\section{Thrombolysis for acute ischaemic stroke: consumer involvement in design of new randomised controlled trial}

Liedeke Koops, Richard I Lindley

\begin{abstract}
Objectives To determine whether consumer involvement would help to solve some of the ethical problems associated with research into thrombolysis for acute ischaemic stroke, with its inherent risk of fatal intracranial haemorrhage.

Design Quantitative and qualitative research. Setting and participants Consultation phase: three meetings were held to discuss the planned research, and participants completed a questionnaire. Qualitative work: focus group meetings explored the issues raised during the consultation phase. Design of information leaflets for patients and relatives: trial materials were drafted during the consultation phase and revised in the light of feedback from the focus group meetings and review by patients and carers on a stroke rehabilitation unit.

Results 54 people attended the consultation meetings. Four (9\%) participants considered the risks of thrombolysis too great, but most (89\%) were prepared to accept the treatment in a clinical trial. Nearly all would accept treatment if it was shown to be effective. Most (85\%) would give their consent to enter the planned trial. The focus group meetings and feedback from patients and carers led to significant changes in the information leaflets. The revised trial materials were considered ethical by a national multicentre research ethics committee.

Conclusions Consumers generally supported a planned trial, and their involvement helped to refine trial consent procedures and led to an ethically acceptable trial design.
\end{abstract}

\section{Introduction}

Thrombolytic treatment for acute ischaemic stroke seems to be beneficial for highly selected patients treated in specialised centres within three hours of the onset of stroke. ${ }^{1}$ In 1997 a systematic review of all randomised controlled trials of thrombolytic treatment for stroke indicated that the benefit might extend to a wider range of patients but confirmed a definite risk of early fatal intracranial haemorrhage. ${ }^{2}$ Only 3435 patients contributed data to this overview, compared with about 60000 patients in trials of thrombolytic treatment for acute myocardial infarction..$^{23}$ As with acute myocardial infarction, a large trial would almost certainly be needed to confirm or refute these results in stroke, but ethical barriers may prevent recruitment in further trials. Patients with acute stroke are often mentally or physically unable to participate in discussions about treatment options. These problems are exacerbated by the time window for effective thrombolytic treatment, which may be just a few hours. Prolonged discussion could delay the start of treatment to the point where treatment is futile. Prompted by the proposal that women should be involved in the design and conduct of research into breast cancer, ${ }^{45}$ we proposed that a similar approach should be tried for stroke medicine. ${ }^{6}$ The early closure of the Australian streptokinase trial and the MAST-Italy trials had illustrated the great concern among stroke physicians about the ethics of performing further studies. Future trials would fail if ethical concerns were not addressed. Our hypothesis was that involving consumers during the design stage would help to solve some of the expected ethical problems.

\section{Methods}

We used a variety of qualitative and quantitative methods to involve consumers. The project involved three main phases: a consultation phase; focus group work; and the development of the consent procedure, supported by information leaflets.

\section{Consultation phase}

One of us (RIL) attended three routine meetings of older people in Edinburgh. These were regular meetings of the Scottish Old Age Pensioners Association Morningside group on 22 September 1997, the Pentland group on 19 November 1997, and the Pentland senior citizens activity club on 6 March 1998. All three meetings followed the same format of a general talk about stroke followed by a discussion about thrombolytic treatment. We used a one page summary of thrombolytic treatment, based on the latest meta-analysis at the time, ${ }^{2}$ to illustrate the potential risks and benefits of treatment. To encourage discussion and document views, we asked people attending the meetings to complete a brief questionnaire (box 1). We emphasised that we were unsure what to do and wanted their opinion.

\section{Focus groups}

We held two focus group meetings to explore the responses obtained during the consultation phase. The
Department of
Clinical
Neurosciences,
University of
Edinburgh, Western
General Hospital,
Edinburgh
EH4 2XU
Liedeke Koops
visiting research
associate
Richard I Lindley
part time senior
lecturer
Correspondence to:
R I Lindley
richard.lindley@
ed.ac.uk
bmj.com 2002;325:415



Patient information sheet on bmj.com 
Box 1: Questionnaire completed by participants in the thrombolysis meetings

- Have you ever had a stroke?

- Do you know someone who has had a stroke?

- Do you think the risks of thrombolytic treatment are too great?

- Would you be prepared to accept these risks in a new trial?

- Would you be prepared to accept these risks if future research confirmed the benefits of the treatment?

- If you had a stroke tomorrow, would you be happy to be included in a clinical trial testing thrombolytic treatment?

- Please write any comments here:

first group consisted of volunteers from the initial meetings, and discussion was centred on an initial draft of a trial summary information leaflet. The second focus group consisted of new volunteers (Bingham and district older people's project), and discussion was focused on the ethical dilemmas of getting consent for stroke trials. We provided refreshments and reimbursed travel expenses; participants were not paid. RIL and LK directed the groups. We recorded the focus group meetings with a pressure zone microphone (Optimus Omnidirectional Boundary Microphone 33-3020) and a tape recorder (Philips Cassette recorder AQ6455). The recording was transcribed using a Sanyo Transcribing System TA 8080. The two authors independently analysed the transcripts to identify emergent themes.

Development of consent procedure and drafting of information leaflets

We presented the results from the first two phases to our research group, and the trial management committee designed a strategy for the consent procedure and revised the information leaflets for patients and relatives. We then distributed the revised leaflets to six patients and carers on our stroke rehabilitation unit for comments. We selected patients who could read and communicate normally and were available during one afternoon session. The information leaflets were revised again after their feedback. The trial management team then finalised the proposed trial procedures and submitted an application to the Scottish multicentre research ethics committee on behalf of all UK centres in 1999.

\section{Results}

Consultation phase

A total of 54 people attended the three meetings. Three $(6 \%)$ participants had previously had a stroke, and most (39/53, 74\%) knew someone who had had a stroke. Four of 47 (9\%) participants considered the risks of thrombolytic treatment to be too great, but most $(42 / 47,89 \%)$ were prepared to accept the risks of treatment in a clinical trial. All but one (98\%) of the 49 participants who responded to the questionnaire were prepared to accept the risks of thrombolytic treatment if future research confirmed its benefit. Forty one of 48 $(85 \%)$ respondents said that they would consent to a randomised controlled trial of thrombolytic treatment if they had a stroke tomorrow. Box 2 gives some of the comments.

\section{Focus group results}

The first focus group was attended by nine people. The second focus group was attended by 10 older people plus one younger facilitator. Box 3 shows the nine main themes identified.

Participants were aware of disability from stroke and gave examples from their own experience: "My sister had a stroke ... when she was in her forties, and she was really bad," "I took a stroke two years ago ... and I was paralysed right down the one side, my face dropped ... and what I am left with now is a weakness in my right hand and a slight limp," "Well I thought I was speaking alright, but my daughter said it was like a bairn [baby] babbling a lot of rubbish." Discussion supported the notion that these experiences of stroke were recognised by most participants.

Participants expressed a wide range of views about the way treatment should be chosen. Some would accept whatever was given. People recognised that clinical trials involving patients were needed: "You can

Box 2: Comments from participants in the thrombolysis meetings

- "I find this very difficult to answer. I realise that research is important, but do not feel qualified enough to accept whether the risks are too great or are not too great."

- "Carry a card with consent. Let relatives know you agree with the treatment. People at high risk for stroke should be informed about a choice of treatment beforehand."

- "I would prefer to carry a card so that I could make my own decision while I was able"

- "At my age I'd rather take a risk in the hope of retaining my independence."

- "All for research as long as patients benefit."

- "Thanks for the advice"

- "It is very encouraging to find a doctor willing to consult the public in this way!"

- "I shall have to think more about this, as I haven't heard about it."

- "Sincerely hope I continue to keep the good health I enjoy just now, but hope your treatment would bring me comfort should I need it."

- "I hope that the clot busters are being used if I should ever have a stroke."

Box 3: Common themes to emerge from consumers during focus group meetings

- Knowledge of disability from stroke

- Knowledge about treatments for stroke and treatment choice

- Taking risks in life

- Acceptability of next of kin giving assent

- Trusting the doctor and potential for abuse

- Acknowledgment of the ethical dilemmas

- Suggesting an advance directive or card

- Accepting informed consent as a good thing

- Wanting precise information on risks and benefits 
try drugs till you're blue in the face on animals, but animals ... not a human person." Knowledge about trials came from television and newspapers: "There's drugs in one pill and a placebo in another." One person did not like the idea of randomisation and wanted to choose the treatment.

Many participants were comfortable with the concept of risk. The risk of a fatal intracranial bleed with thrombolysis was mentioned, and some people commented: "Four people in 100 is a very small risk compared to living a vegetable life," "I think at my age I have nothing to lose," "The quality of life is what matters," "I have made a living will, but if I should become disabled through accident or injury ... I just want to be given pain killers and allowed to die peacefully." One person thought that older people should not be included: "I think it would be very unfair to try things out on someone who has reached eighty." Another participant commented that "I would not want to sacrifice one life to save mine." Participants recognised risks in other areas of medicine: "Even if you have a comparatively simple operation, which is done every day of the week, there is a statistical and finite chance of this failing." Many people accepted the risks due to the play of chance: "It's like Russian Roulette, isn't it?", "If you got to go, you're gonna go." One group had a discussion about the maximum average risk they would be prepared to accept for a treatment that may prevent disability, and they thought a risk of up to $20 \%$ of immediate death was acceptable.

In the case of a patient unable to communicate, participants were unanimous that the next of kin was the appropriate person to decide on treatment. However, some people worried about the consequences of this: "The implications of that though are think of the guilt that might put on someone signing and then the person died and they were aware they had been party to doing that," "I would not like to put them in that situation." If the next of kin could not be contacted, assent by the attending doctor was considered a possible alternative: "It's up to the doctor," "You should use your discretion. And if you think it's going to work, go for it!" However, they recognised the potential risks to this strategy: "There are foolish people in every profession," "But actually you could really abuse people that are already vulnerable because they have not got anyone else that is fighting for them to say, no I don't want that to happen, so you could deliberately use people who have no relatives." One person thought that doctors should simply give the treatment rather than "experiment" on them.

Participants recognised the ethical dilemma involved in obtaining valid consent: "The doctor's in a very, very difficult position," "When you go in with a stroke you can't honestly be saying that you can make a clear rational decision when you are lying there." The groups considered some solutions and wondered whether an advance directive could be useful or whether people should people carry cards to confirm that they would consent to emergency treatment as part of a randomised controlled trial.

One group discussed the concept of informed consent and agreed that this was a good idea: "Different natures react differently, to all sorts of information, especially about health." In general, the groups disliked adjectives such as "large," "small," or "massive" and pre- ferred to have risks explained in percentages or proportions. Overall, the groups generally agreed that the research seemed worthwhile, and they were pleased to have been consulted.

\section{Development of information leaflets and consent procedure}

We edited the leaflets to remove adjectives such as "small" risk and "massive" bleeding and incorporated approximate percentage risks derived from the latest Cochrane review. We wrote a version suitable for patients and one for carers. We gave these leaflets to six selected carers and patients on the stroke rehabilitation unit. The patients had few comments and would have been happy to take the risk of thrombolysis if it could have prevented their current disability. The carers thought the leaflets inadequately described the consequences of severe stroke (most of their relatives had severe disability).

The trial management team revised the leaflets again, incorporating a staged consent procedure allowing for differing individual circumstances: (1) Patients are able to sign a consent form. (2) Patients could provide verbal consent (which could be witnessed). (3) Assent by a relative for patients unable to give consent themselves. (4) Waiver of consent, following strict guidelines adapted from the United States.

We then submitted this final consent procedure to the multicentre research ethics committee. The committee asked for additional changes to incorporate a lay person's description of the uncertainty principle and a few administrative changes. These final changes were then accepted in November 1999 (see web extra for final version of information sheet), and full ethics approval was granted, including a procedure for waiver of consent.

\section{Discussion}

The quantitative survey provided general support for the trial, although $9 \%$ of participants were worried about the risks of thrombolysis. All but one participant would have been prepared to accept the risks if treatment was shown to be effective. Participants recognised the ethical dilemmas.

The focus group participants also recognised the ethical dilemmas but were able to suggest solutions. These included being more specific about the potential risks and benefits, recognising the importance of discussing the trial with the next of kin (if the patient was mentally incompetent as a result of the stroke), and suggesting that the doctors may have a role in a consent procedure if relatives were not available ("waiver of consent").

We made many changes to our leaflets as a result of this consultation process. For the patients (and their relatives) these documents are the only written record of the trial and are thus very important. Most ethics committees spend more time advising of the need for appropriate information leaflets than on any other aspect of trial design. We recommend that future consumer involvement should include patients (and their carers) with the index medical condition. ${ }^{7}$ Overall, we found that our consumers understood complex ethical 
issues, provided thoughtful comments, and generated interesting discussion.

Our research can be criticised for several weaknesses. Firstly, our consumers were highly selected. Secondly, one of us (RIL) is the lead researcher for the trial and may have unduly influenced the participants. We were aware of this potential bias and made great efforts to allow discussion by the groups, only intervening to bring the discussion back to the subject.

Although consumers have been involved in the design of randomised controlled trials in HIV, women's health, and neonatal medicine, ${ }^{8}$ consumer involvement in randomised controlled trials on stroke has not been reported before. Our results are important for several reasons. Firstly, our trial involves a particularly risky treatment. We have established that this type of risk is surprisingly acceptable, in the context of the risks of the natural history of stroke. This concept is generally accepted in legal terms as "minimal risk"-that is, the risks of treatment, in the context of the risks facing untreated patients, are relatively minimal. The acceptability of risks in stroke medicine has been reported previously. ${ }^{910}$ Secondly, despite the potential ethical barriers to our planned trial, our trial material was accepted after only one cycle of amendments with the national ethics committee, an important achievement.

American guidelines for a procedure for waiver of consent recommend public consultation, and we support these recommendations. ${ }^{11}$ We believe that medical researchers-and the general public-have a responsibility to develop and refine medical ethics, and that this should not be left wholly to professional medical ethicists. We believe that the acceptance of a "waiver of consent" would be an advance for emergency stroke research in the United Kingdom (and elsewhere). However, the new Adults with Incapacity Act in Scotland has made waiver of consent illegal for trials of emergency treatment despite lobbying by many in the Scottish medical profession.

Our final comment is addressed to other researchers planning randomised controlled trials. Our groups of older people valued the opportunity to discuss medical issues with a local clinician, and they all seemed to enjoy the meetings. Overall, we found this a very positive experience.

We thank the participants from the Pentland and Morningside Scottish Old Age Pensioners Association groups, the Pentland senior citizens activity group, Bingham and district older people's project, and patients and carers from the stroke rehabilitation unit of the Royal Victoria Hospital, Edinburgh. Senga Bond, John Forbes, and Sally Wyke provided valuable advice on how to organise and run focus groups. Charles Warlow, Peter Sandercock, and Joanna Wardlaw provided valuable comments on the manuscript. The ideas of Hazel Thornton, Michael Baum, and Iain Chalmers prompted this research.

Contributors: LK designed and ran the focus groups, analysed the meeting transcripts, and co-wrote the paper. RIL devised the study, ran the initial consultation phase, independently analysed the tape transcripts, and co-wrote the paper. $\mathrm{He}$ acts as guarantor for the work.

\section{What is already known on this topic}

Obtaining informed consent for emergency stroke treatment is difficult and presents many ethical dilemmas

Consumer involvement in trial design has been successful in some medical specialties but not previously reported in stroke research

\section{What this study adds}

Involvement of consumers in the design of trials on stroke is valuable

Comments from people who have not yet had a stroke and from carers of those who have can enable substantial improvement of trial information leaflets

Consumers support different consent approaches depending on the clinical state of the patient

Consumer involvement can be a very important part of the development of new randomised controlled trials

Funding: Boehringer Ingelheim provided an unrestricted educational grant to support this work but had no part in the design, conduct, analysis, or reporting of the study.

Competing interests: RIL has accepted honoraria for lectures at satellite symposia sponsored by Boehringer Ingelheim and is supported in attending stroke conferences by Boehringer Ingelheim for his role as editor of Stroke Matters (1997 to present). Boehringer Ingelheim holds the European licence for alteplase, a thrombolytic agent, and has donated a supply of alteplase and placebo for the third international stroke trial, an independent trial evaluating alteplase for acute ischaemic stroke, of which RIL is the principal investigator.

1 National Institute of Neurological Disorders and Stroke rt-PA Stroke Study Group. Tissue plasminogen activator for acute ischemic stroke. $N$ EnglJ Med 1995;333:1581-7.

2 Wardlaw JM, Warlow CP, Counsell C. Systematic review of evidence on thrombolytic therapy for acute ischaemic stroke. Lancet 1997;350:607-14.

3 Fibrinolytic Therapy Trialists' (FTT) Collaborative Group. Indications for fibrinolytic therapy in suspected acute myocardial infarction: collaborative overview of early mortality and major morbidity results from all randomised trials of more than 1000 patients. Lancet 1994;343:311-22.

4 Thornton H. Clinical trials: a "ladyplan" for trial recruitment? - everyone's business! Lancet 1993;341:795-6.

5 Baum M. New approach for recruitment into randomised controlled trials. Lancet 1993;341:812-3.

6 Lindley RI. Thrombolytic treatment for acute ischaemic stroke: consent can be ethical. BMJ 1998;316:1005-7.

7 Entwistle VA, Renfrew MJ, Yearley S, Forrester J, Lamont T. Lay perspectives: advantages for health research. BMJ 1998;316:463-6.

8 Hanley B, Truesdale A, King A, Elbourne D, Chalmers I. Involving consumers in designing, conducting, and interpreting randomised controlled trials: questionnaire survey. BMJ 2001;322:519-23.

9 Gage $\mathrm{BF}$, Cardinalli $\mathrm{AB}$, Owebs $\mathrm{DK}$. The effect of stroke and stroke prophylaxis with aspirin or warfarin on quality of life. Arch Intern Med 1996;156:1829-36

10 Solomon NA, Glick HA, Russo CJ, Lee J. Schulman KA. Patient preferences for stroke outcomes. Stroke 1994;25:1721-5.

11 Wichman A, Sandler AL. Research involving critically ill subjects in emergency circumstances: new regulations, new challenges. Neurology $1997 ; 48: 1151-5$

(Accepted 4 April 2002) 